\title{
Administration of CD34+ cells after stroke enhances neurogenesis via angiogenesis in a mouse model
}

\author{
Akihiko Taguchi, ${ }^{1}$ Toshihiro Soma, ${ }^{2}$ Hidekazu Tanaka, ${ }^{3}$ Takayoshi Kanda, ${ }^{4}$ Hiroyuki Nishimura, ${ }^{5}$ \\ Hiroo Yoshikawa, ${ }^{5}$ Yoshitane Tsukamoto, ${ }^{6}$ Hiroyuki Iso, ${ }^{7}$ Yoshihiro Fujimori, ${ }^{8}$ David M. Stern, ${ }^{9}$ \\ Hiroaki Naritomi, ${ }^{1}$ and Tomohiro Matsuyama ${ }^{5}$

\begin{abstract}
1Department of Cerebrovascular Disease, National Cardiovascular Center, Osaka, Japan. ${ }^{2}$ Department of Hematology, Osaka Minami National Hospital, Osaka, Japan. ${ }^{3}$ Department of Pharmacology, Graduate School of Medicine, Osaka University, Osaka, Japan. ${ }^{4}$ Department of Gynecology, Osaka Minami National Hospital, Osaka, Japan. ${ }^{5}$ Department of Internal Medicine, Hyogo College of Medicine, Hyogo, Japan. ${ }^{6}$ Department of Pathology, Osaka Medical Center for Cancer and Cardiovascular Disease, Osaka, Japan. ${ }^{7}$ Department of Psychology and ${ }^{8}$ Department of Hematology,
\end{abstract} \\ Hyogo College of Medicine, Hyogo, Japan. ${ }^{9}$ Medical College of Georgia, Augusta, Georgia, USA.
}

\begin{abstract}
Thrombo-occlusive cerebrovascular disease resulting in stroke and permanent neuronal loss is an important cause of morbidity and mortality. Because of the unique properties of cerebral vasculature and the limited reparative capability of neuronal tissue, it has been difficult to devise effective neuroprotective therapies in cerebral ischemia. Our results demonstrate that systemic administration of human cord blood-derived CD34 ${ }^{+}$ cells to immunocompromised mice subjected to stroke 48 hours earlier induces neovascularization in the ischemic zone and provides a favorable environment for neuronal regeneration. Endogenous neurogenesis, suppressed by an antiangiogenic agent, is accelerated as a result of enhanced migration of neuronal progenitor cells to the damaged area, followed by their maturation and functional recovery. Our data suggest an essential role for $\mathrm{CD}^{+} 4^{+}$cells in promoting directly or indirectly an environment conducive to neovascularization of ischemic brain so that neuronal regeneration can proceed.
\end{abstract}

\section{Introduction}

Thrombo-occlusive atherosclerotic cardiovascular disease is a major cause of death and disability in developed countries. In the acute phase, therapeutic maneuvers include fibrinolytic therapy to restore blood flow to the ischemic site. In the longer term, formation of new blood vessels is necessary to fully supply tissue metabolic and functional requirements. Although it had been assumed that postnatal development of neovessels resulted only from outgrowth of pre-existing vasculature, it has become evident that circulating endothelial progenitor cells (EPCs), contained in a CD $34^{+}$cell population enriched in cord blood, have the capacity to participate in neovascularization of ischemic tissues $(1,2)$. Thus, a new strategy proposed for enhancing recovery due to ischemic stress is administration of EPCs to stimulate formation of neovasculature. In this context, recent reports have demonstrated that infusion of EPCs results in their incorporation into neovasculature at the ischemic site and limitation of tissue damage in animal models (3). Furthermore, human $\mathrm{CD} 34^{+}$cells were shown to secrete numerous angiogenic factors, including VEGF, HGF, and IGF-1 (4). On the basis of these observations, clinical trials of cell transplantation in hindlimb $(5,6)$ and cardiac ischemia (7) have been initiated with promising results.

Nonstandard abbreviations used: anterior cerebral artery (ACA); cerebral blood flow (CBF); chloromethylbenzamido (CM-DiI); doublecortin (DCX); endothelial progenitor cell (EPC); erythropoietin (EPO); fetal liver kinase-1 (Flk-1); high-power field (HPF); middle cerebral artery (MCA); neuronal progenitor cell (NPC); neuron-specific nuclear protein (NeuN); phycoerythrin (PE); polysialylated neuronal cell adhesion molecule (PSA-NCAM); subventricular zone (SVZ); 2,3,5-triphenyltetrazolium (TTC). Conflict of interest: The authors have declared that no conflict of interest exists.

Citation for this article: J. Clin. Invest. 114:330-338 (2004).

doi:10.1172/JCI200420622
Stroke is another setting of occlusive thromboatherosclerotic disease in which acceleration of angiogenesis might be expected to enhance the outcome. Despite the improvement of poststroke neurological outcome by administration of human cord blood cells (8) or bone marrow-derived cells (9) (both potentially a rich sources of stem cells including $\mathrm{CD}_{3} 4^{+}$cells) in rodent models, few of the administered cells could be demonstrated in brain parenchyma expressing neuronal markers, raising a question as to the underlying mechanism. The results of our study demonstrate that systemic administration of human $\mathrm{CD} 4^{+}$cells to immunocompromised mice subjected to stroke 48 hours earlier accelerates neovascularization of the ischemic zone. Such a rich vascular environment, along with generation of other nurturing neuronal mediators by CD34 ${ }^{+}$ cells, such as VEGF, FGF2, and IGF-1 (10-12), enhances subsequent neuronal regeneration; endogenous neurogenesis is accelerated as neuronal progenitors migrate to the damaged area, followed by their maturation and survival when $\mathrm{CD} 34^{+}$cells have stimulated the formation of increased vascular channels. In contrast, in the presence of an antiangiogenic agent, the beneficial effect of CD34 ${ }^{+}$cells was lost. Our results provide the first direct link between vasculogenesis and neurogenesis in the repair of ischemic brain lesions.

\section{Results}

Induction of stroke and proliferation of endothelial cells in situ. A reproducible model of stroke in the middle cerebral artery (MCA) cortex, sparing the striatum, was developed in SCID mice by permanent ligation of the M1 distal portion of the left MCA. Subsequent infusion of carbon black showed strongly decreased staining in the affected area. Nonviability of affected tissue was confirmed by 2,3,5-triphenyltetrazolium (TTC) staining. Values of cortical width index (see Methods section) were highly reproducible $(\sim 0.34-0.36)$ 

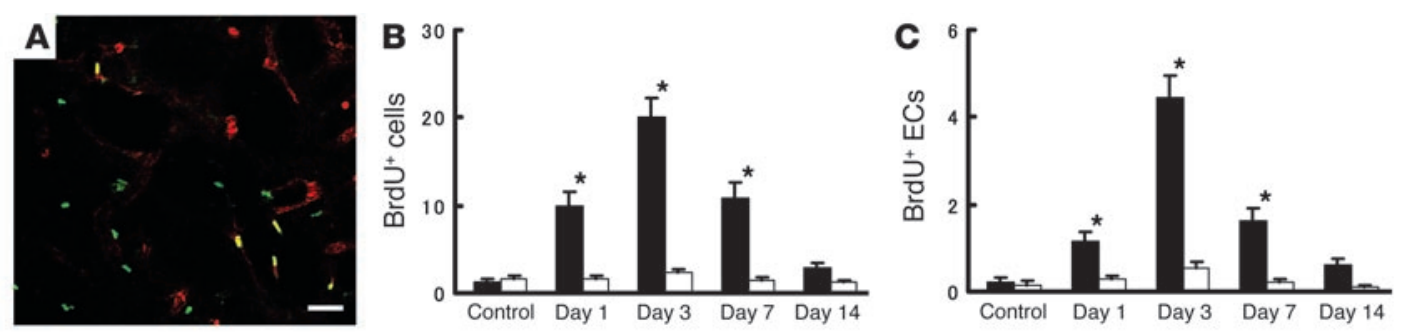

\section{Figure 1}

Endothelial proliferation in situ after stroke. On days 1, 3, 7, and 14 after stroke, the number of proliferating cells (BrdU+) and proliferating endothelial cells (co-staining for BrdU and CD31) was determined in the left cortical area of 1-1.5 mm distal from the midline. (A) Immunohistological analysis of proliferating cells labeled with BrdU (green), anti-mouse CD31 IgG (red), and both (yellow). The number of cells visualized with $\mathrm{BrdU}(\mathbf{B})$ and the subpopulation BrdU+ cells also displaying mouse CD31 (i.e., double positives) (C) are shown. Ten HPFs were evaluated for each animal ( $n=6$ per group) by two investigators blinded to the experimental protocol. Note in C, cells displaying mouse CD31 are termed endothelial cells (ECs). Black bars, ipsilateral; white bars, contralateral. ${ }^{\star} P<0.05$ versus control. Scale bar: $30 \mu \mathrm{m}$.

over the 12-week experimental period. Survival in this stroke model was greater than $95 \%$, and no seizures were observed.

To estimate the optimal time to administer human $\mathrm{CD} 34^{+}$cells, proliferation of endothelial cells in vasculature of the penumbral region (at the leading edge of viable tissue) was assessed by in vivo BrdU labeling. Sections were visualized with antibody to BrdU and mouse-specific antibody to CD31 by confocal microscopy. Cellular profiles co-staining for both markers were considered proliferating endothelial cells (Figure 1A). On days 1 and 3 after stroke, a subpopulation of $\mathrm{BrdU}^{+}$cells also stained with mouse CD31, indicating an endothelial origin of this signal (Figure 1, B and C). By day 7 , although endothelial proliferation continued, it had begun to decrease. In contrast, BrdU-labeled cells were present in a constant, small amount on the contralateral (nonstroke) side (Figure 1, B and C). These data indicated that administration of $\mathrm{CD} 34^{+}$cells on day 2 after stroke would buttress the endogenous proliferative component of the vascular response to cerebral ischemia.

Administration of $\mathrm{CD} 34^{+}$cells after stroke. Human CD34+ cells $(95 \%$ pure $\mathrm{CD} 4^{+}$cells) isolated from human cord blood or control cells (CD34- cells with $<0.2 \% \mathrm{CD}^{-} 4^{+}$cells, also from human cord blood) were administered intravenously via tail vein 48 hours after stroke. Analysis of cell surface markers revealed that $1.5 \% \pm 0.1 \%$ and $0.9 \% \pm 0.1 \%$ of the $\mathrm{CD} 34^{+}$cell population expressed the endothelial lineage markers fetal liver kinase-1 (Flk-1) (1) and P1H12 (13), respectively $(n=4)$. The effect of CD $34^{+}$cells was evident within 24 hours of their transplantation. Labeling vasculature by infusion of carbon black ink demonstrated neovasculature at the border of the MCA and anterior cerebral artery (ACA) cortex (staining with TTC demarcates viable and nonviable tissue) in animals treated with $\mathrm{CD} 34^{+}$cells (Figure 2, A and B), compared with those receiving CD34- cells (Figure 2C) or PBS alone (Figure 2D). Determination of the angiographic score confirmed the impression of increased neovasculature in animals transplanted with $\mathrm{CD}^{+} 4^{+}$cells, compared with other groups (Figure 2E). To evaluate vascular activation in affected cerebral vessels, we used mouse-specific antibody to CD13, an antigen expressed by endothelial cells in angiogenic, but not quiescent, vasculature (14). Visualization of mouse CD13 in brain sections 24 hours after cell transplantation showed that cells bearing this activated endothelial marker were most evident in sections from mice treated with $\mathrm{CD} 34^{+}$ (Figure 2F), compared with those receiving CD34- cells (Figure 2G) or PBS (Figure $2 \mathrm{H}$ ). Increased density of vasculature in the ischemic territory of animals treated with $\mathrm{CD} 34^{+}$cells translated to significantly enhanced cerebral blood flow (CBF) (Figure 2I).
To analyze the effect of subpopulations within the general CD34 cell population, we compared the effect of poststroke transplantation of the same number of $\mathrm{CD} 4^{+}$cells (containing Flk-1- ${ }^{-}$and Flk- $1^{+}$cells) with $\mathrm{CD} 34^{+} / \mathrm{Flk}-1^{-}$cells on vascular activation and neovascularization. Brain tissue was examined 7 days after cell transplantation, because EPCs are known to incorporate into capillary walls at ischemic sites by this time point after the ischemic episode (2). FACS analysis confirmed that the CD34 ${ }^{+} / \mathrm{Flk}^{-} 1^{-}$ population contained less than $0.1 \%$ Flk- $1^{+}$cells $(n=4)$. On the basis of CD13 staining (using the same mouse-specific antibody mentioned earlier), there was similar activation of endogenous endothelium after transplantation of either CD $34^{+}$cells (including both Flk-1- and Flk-1 ${ }^{+}$ subpopulations) and CD34 $4^{+}$Flk-1- cells (not shown). Although neovasculature was observed at the border of the MCA and ACA cortex in animals treated with CD34+ cells (Figure $2 \mathrm{~J}$ ) and CD $34^{+} / \mathrm{Flk}-1^{-}$cells (Figure $2 \mathrm{~K}$ ), mice treated with $\mathrm{CD} 34^{+}$cells displayed increased neovascularization based on angiographic score (scores of $22 \pm 3$ and $13 \pm 2$, for $\mathrm{CD} 4^{+}$and $\mathrm{CD} 34^{+} /$Flk- $1^{-}$cells, respectively; $P<0.05, n=6$ ).

Transplantation of $\mathrm{CD} 34^{+}$cells and poststroke functional recovery. Stroke causes motor deficits and behavioral abnormalities (15). Dysfunction of the cortex is closely linked to disinhibition of behavior (16). Compared with sham-operated controls, mice that received CD34cells or PBS displayed significant behavioral abnormalities on day 90 after cell transplantation ( $n=12$, for each group). Rearing counts under lighted conditions were $8.4 \pm 0.8$ (PBS), $8.7 \pm 0.5$ (CD34- cells), $4.2 \pm 0.5$ (CD34 ${ }^{+}$cells), and $3.0 \pm 0.5$ (sham-operated controls) for each of the groups. Counts of locomotion were $5.1 \pm 0.4$ (PBS), $5.0 \pm 0.4$ (CD34- cells), $3.7 \pm 0.4$ (CD34+ cells), and $3.3 \pm 0.4$ (sham). ANOVA revealed hyperactivity with respect to both rearing $(P<0.01)$ and locomotion $(P<0.01)$ in the CD34- cell- and PBS-treated groups, compared with sham-operated controls. In contrast, mice treated with $\mathrm{CD} 34^{+}$cells showed no significant hyperactivity compared with sham-operated controls $(P>0.05)$, and displayed significant improvement in both behavioral tests compared with animals that received PBS or CD34- cells $(P<0.05)$. Mice treated with CD34- cells or PBS after stroke showed loss of this "dark" response, with respect to rearing $(P>0.05)$ and locomotion $(P>0.05)$. In contrast, animals treated with CD34 ${ }^{+}$cells displayed the expected increase in rearing and locomotion in the darkness $(P<0.01)$.

Using another behavioral paradigm, excessive startle consequent to auditory stimulation was observed in poststroke animals treated with CD34- cells and PBS. Startle amplitudes were $0.9 \pm 0.1$ volts (PBS), $0.8 \pm 0.1$ (CD34- cells), $0.5 \pm 0.1$ (CD34 $^{+}$ 

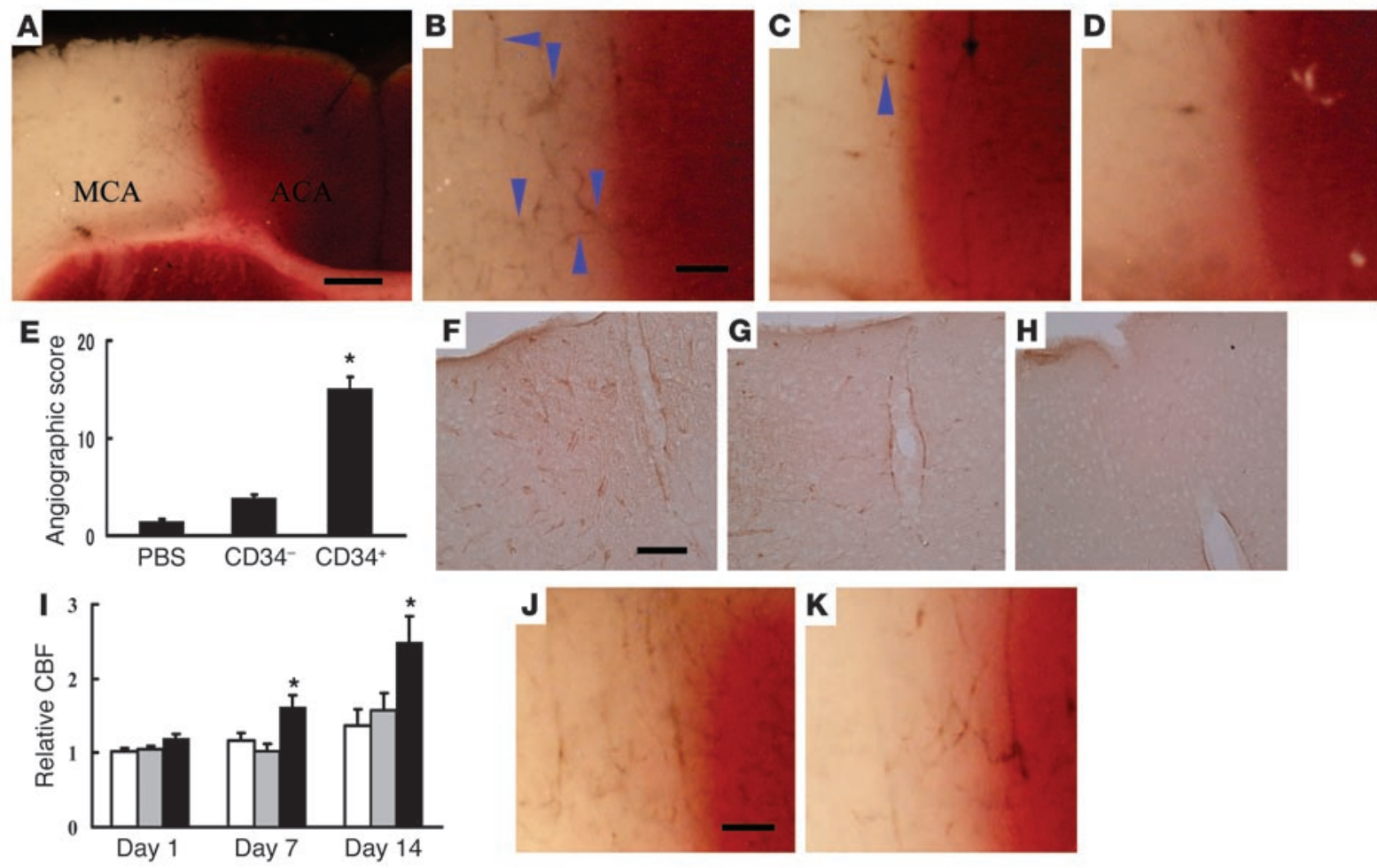

Figure 2

Transplantation of $\mathrm{CD}_{4} 4^{+}$cells after stroke accelerates neovascularization. (A-D) Mice subjected to stroke received CD34+ cells (A and B), CD34- cells (C), or PBS alone (D) on day 2. Animals were infused with carbon black ink and killed at 24 hours after cell transplantations. Sections were stained with TTC. Neovascularization was noted at the border zone between the ACA and MCA areas (arrowheads show microvessels), especially in animals treated with CD34+ cells compared with those receiving CD34- cells or PBS alone. (E) An angiographic score for each experimental condition based on analysis of 6 mice per group. $(\mathbf{F}-\mathbf{H})$ Activated endothelial cells were observed with antibody specific for mouse CD13 in the ACA area. F: CD34+ cells; G: CD34- cells; H: PBS. (I) CBF was measured in the MCA area just outside of the penumbra, and values in animals treated with CD34+ cells (black bars), CD34- cells (gray bars), or PBS (white bars) were compared with values before cell transplantation at times corresponding to days 1,7 , and 14 after cell transplantation ( $n=6$ per group). Data shown are relative CBF versus day the measurement was performed. ( $\mathbf{J}$ and $\mathbf{K}$ ) Labeling vasculature by infusion of carbon black ink demonstrated neovasculature at the border of the MCA and ACA cortex in animals treated with CD34+ cells $(\mathbf{J})$ and CD34+/Flk-1- cells $(\mathbf{K})$ on day 7 after cell transplantation. Scale bars: 0.5 $\mathrm{mm}(\mathbf{A})$ and $0.1 \mathrm{~mm}(\mathbf{B}, \mathbf{F}$, and $\mathbf{J}) .{ }^{*} P<0.05$ versus $\mathrm{PBS}$.

cells), and $0.4 \pm 0.1$ (sham) on day 90. ANOVA revealed hyperactivity in the CD34- cell- and PBS-treated groups, compared with sham-operated controls $(P<0.05)$. In contrast, mice treated with $\mathrm{CD} 34^{+}$cells showed no significant hyperactivity compared with sham-operated controls $(P>0.05)$. Shortening of the latency period with fear conditioning was also observed in poststroke animals treated with CD34- cells and PBS compared with shamoperated controls $(P<0.05)$. In contrast, mice treated with CD34 ${ }^{+}$ cells showed no significant hyperactivity $(P>0.05)$. Similar behavioral abnormalities were also observed on day 35 after cell transplantation. Since ischemic injury in our stroke model was limited to the cortex (hippocampus and striatum were intact), it was not surprising that spatial learning (water maze testing) and passive avoidance remained unchanged and comparable in all groups when assessed 5 weeks after stroke (data not shown). Mice showed rapid recovery from focal motor deficits, and by day 16 after stroke no motor deficits were detected using a modified three-point scale (17) (data not shown).

Effect of poststroke $\mathrm{CD} 34^{+}$cell transplantation on endogenous neurogenesis and cortical expansion. These results suggested that cortical function and neoangiogenesis, potentially supporting integrity of the MCA cortex, might be better maintained in animals treated with $\mathrm{CD} 34^{+}$ cells after stroke. Such maintenance of cortical integrity might reflect $\mathrm{CD}_{3} 4^{+}$cell-mediated enhancement of the survival of ischemic neurons or acceleration of endogenous neurogenesis. Representative brains from poststroke animals in each group (treated with PBS alone, CD34- cells, or CD34+ cells) are shown in Figure $3, \mathrm{~A}-\mathrm{F}$. There was a prominent increase in cortical width index in animals receiving $\mathrm{CD} 34^{+}$cells at 14,35 , and 90 days after cell transplantation (Figure 3G). Thus, cortical expansion in animals treated with $\mathrm{CD} 34^{+}$cells was most likely to reflect the result of ongoing neurogenesis, rather than maintenance of the integrity of ischemic neurons from the time of induction of stroke.

Cell trace analysis with a fluorescent dye revealed that labeled transplanted $\mathrm{CD} 34^{+}$cells were not co-stained with antibody to a neuronal (neuron-specific nuclear protein; NeuN) or an astrocyte (glial fibrillary acidic protein) marker (not shown). However, activation of endogenous neurogenesis was observed after stroke, and this was accentuated in mice receiving $\mathrm{CD} 34^{+}$cells. On day 7 after administration of $\mathrm{CD} 34^{+}$cells, cells expressing the polysialylated neuronal cell adhesion molecule (PSA-NCAM), a marker of migrating neuronal progenitor cells (NPCs) (18), were observed in the subventricular zone (SVZ), and migration of these NPCs toward the ischemic zone was visualized on day 14 using markers 

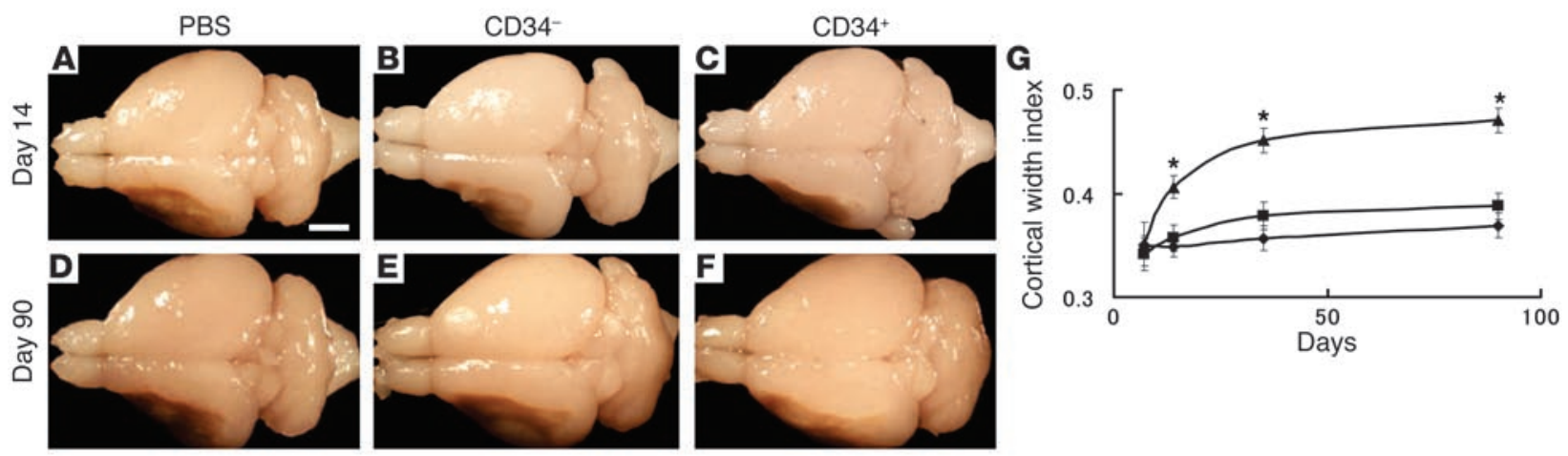

Figure 3

CD34+ cell transplantation induces cortical expansion after stroke. (A-F) On day $14(\mathbf{A}-\mathbf{C})$, and day 90 (D-F) after cell transplantation, the brains of mice were evaluated grossly. Compared with poststroke mice treated with PBS (A and $\mathbf{D})$ or infused with CD34- cells (B and E), animals transplanted with $\mathrm{CD}_{3} 4^{+}$cells ( $\mathbf{C}$ and $\mathbf{F}$ ) showed an increase in area occupied by the left cortex. (G) Cortical regeneration was induced by $\mathrm{CD}_{34}{ }^{+}$cells transplantation: triangles, CD34+ cells; squares, CD34- cells; diamonds, PBS. In each case, there were 6 animals per group. Scale bar: 2 mm (A). ${ }^{*} P<0.05$ versus PBS.

for PSA-NCAM (Figure 4, A and B, corresponding to lower and higher magnifications, respectively), as well as the neuronal stem cell marker, Musashi-1 (Figure 4C) (19). Expression of doublecortin (DCX), a marker of immature, or early, neurons (20), was principally restricted to NPCs close to the SVZ (Figure 4D). In contrast, expression of a neuronal cell marker, NeuN, was observed after cell migration from the SVZ and appeared as a signal of lower intensity compared with nearby mature neurons (Figure 4E). The situation was quite different on the contralateral side in poststroke animals treated with $\mathrm{CD}_{3} 4^{+}$cells; no such movement of precursors (PSA-NCAM ${ }^{+}$cells) was observed (Figure 4F). Although migrating NPCs were also seen in poststroke animals treated with PBS (Figure 4G) and CD34- cells, this finding was considerably magnified in mice receiving $\mathrm{CD}^{+} 4^{+}$cells (Figure 4H).

To investigate mechanisms underlying cortical expansion, immunohistological analysis was conducted to visualize PSA-NCAM, NeuN, and MAP-2. On day 14 after transplantation of CD34- cells after stroke, mature cortical neurons displaying neuronal markers NeuN (Figure 5A) and MAP-2 (Figure 5B) were observed up to the periphery of the ischemic area, whereas only a thin layer of migrating PSA-NCAM ${ }^{+}$ NPCs was observed at the ischemic edge (Figure 5C). In contrast, transplantation of $\mathrm{CD} 34^{+}$cells after stroke expanded cortical areas displaying a low density of $\mathrm{NeuN}^{+}$(Figure 5D) and MAP-2 $2^{+}$cells (Figure 5E) beyond the boundary demarcating mature neurons. Migration of NPCs into this expanded area was also observed by PSA-NCAM staining (Figure 5F).

Next, we investigated the fate of NPCs migrating from the SVZ to the ischemic area. Poststroke animals treated with CD34- cells displayed many cells with $\mathrm{TUNEL}^{+}$nuclei around the lower part of cortical ischemic edge (Figure 5G). In contrast, there were a minimal number of $\mathrm{TUNEL}^{+}$nuclei observed in the same region in animals subject to transplantation of $\mathrm{CD} 34^{+}$cells after stroke (Figure

\section{Figure 4}

5, $\mathrm{H}$ and I). Thus, animals treated with $\mathrm{CD} 34^{+}$cells after stroke display migration and survival of NPCs, which eventually contribute to a population of more mature neurons.

To confirm this evidence of neurogenesis, animals transplanted with $\mathrm{CD}^{+} 4^{+}$cells, CD34- cells, or PBS after stroke were infused with
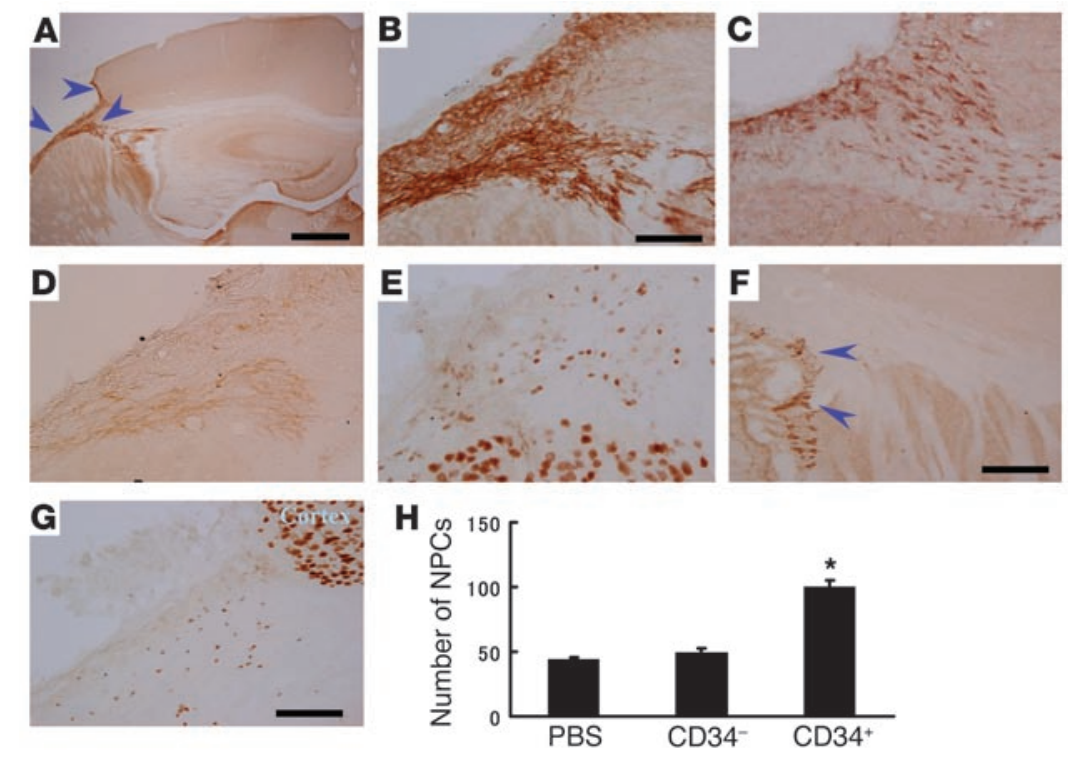

CD34+ cell transplantation accelerates neuronal regeneration after stroke. (A and B) On day 14, animals receiving CD34+ cells after stroke displayed migration of NPCs toward the ischemic area by PSA-NCAM immunostaining. (C-E) Analysis of serial sections displayed expression of neuronal stem cell markers, Musashi-1 (C) and DCX (D). Note that expression of DCX was limited to the area proximal to the SVZ. PSA-NCAM+ NPCs also expressed NeuN (E). Small NeuN ${ }^{+}$nuclei were observed in PSA-NCAM+ NPCs, whereas more intensely staining and larger nuclei represent mature neurons. (F) On day 14 on the contralateral side, PSA-NCAM+ NPCs were limited to the SVZ; that is, no migration of NPCs was observed. (G) Migration of NPCs with small NeuN+ nuclei toward cortex was observed in poststroke mice treated with PBS on day 14 after cell transplantation. (H) The average number of NPCs in the white matter at the lower left of the left cortex per HPF from 5 animals under each condition. Three sections were evaluated in each animal, and $n=5$ per group. Arrowheads delineate individual NPCs or demarcate areas rich in NPCs. Scale bars: $1 \mathrm{~mm}(\mathbf{A}), 0.2 \mathrm{~mm}(\mathbf{B}$ and $\mathbf{F})$, and $0.4 \mathrm{~mm}(\mathbf{G}) .{ }^{*} P<0.05$ versus PBS. 

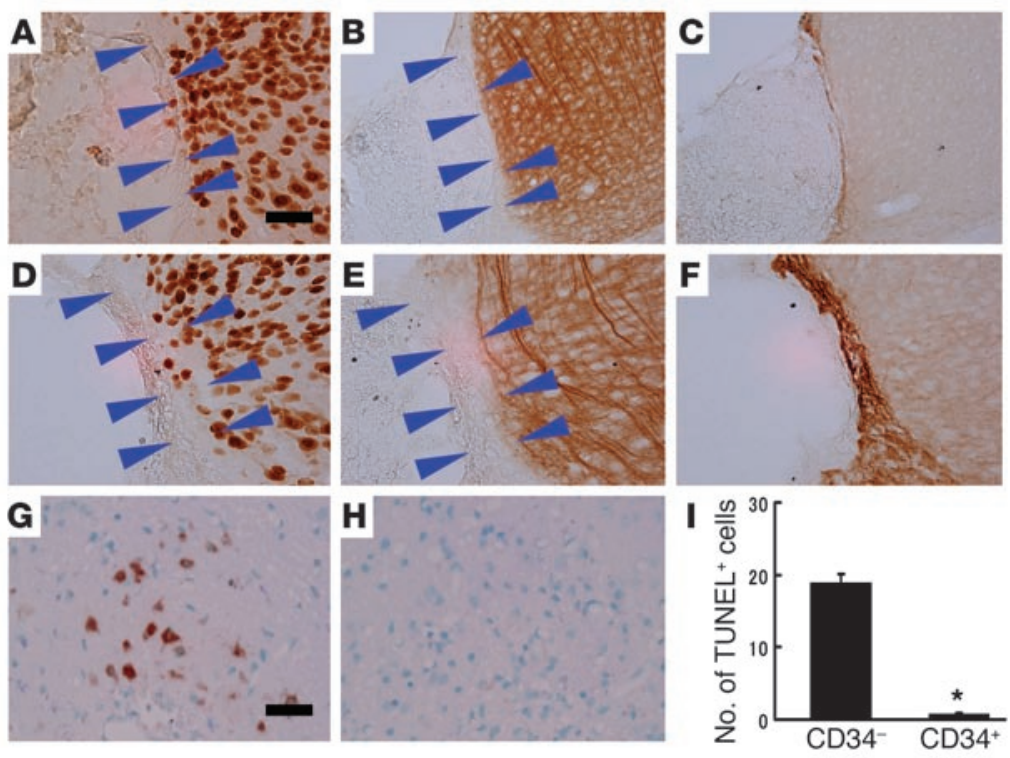

Figure 5

Therapeutic neovascularization, due to $\mathrm{CD} 34^{+}$cell transplantation after stroke, enhances neurogenesis. (A-F) On day 14 after CD34- cell transplantation, mature cortical neurons were observed up to the edge of the ischemic region displaying neuronal markers, NeuN (A) and MAP-2 (B), whereas only a thin layer of migrating PSA-NCAM+ NPCs was observed at the ischemic edge (C). In contrast, after transplantation of CD34+ cells, expanded cortical areas displaying a low density of $\mathrm{NeuN}^{+}$(D) and MAP-2+ cells (E) were observed beyond the boundary demarcating mature neurons. Migration of NPCs into this expanded area was also observed by PSA-NCAM staining (F). (G-I) On day 14 , TUNEL ${ }^{+}$cells were visualized around the lower part of the expanded cortical area. Whereas massive cell death was observed in animals receiving CD34- cell transplantation (G), the number of TUNEL+ profiles was strongly reduced in mice transplanted with CD34+ cells (H). (I) The average number of TUNEL+ cells per HPF. Three sections were evaluated in each animal; $n=5$ per group. Arrowheads indicate the expanded cortical areas displaying a low density of indicated marker. Scale bars: $100 \mu \mathrm{m}(\mathbf{A})$ and $50 \mu \mathrm{m}(\mathbf{G}) .{ }^{\star} P<0.05$ for CD34+ versus CD34- cell transplantation.

BrdU every second day for a total of 14 days and then killed. BrdUand NeuN-bearing cell populations were visualized to determine if a group of neurons might be undergoing cell division. In animals treated with PBS alone (Figure 6A) or CD34- cells (Figure 6B), double staining for BrdU and NeuN displayed little evidence of cells (i.e., nuclei) expressing both markers. Only in animals infused with $\mathrm{CD} 4^{+}$cells after stroke did we consistently observe double-labeled cell profiles with BrdU and NeuN to produce a merged image with cells displaying both markers (Figure 6C). Quantification of these results showed a significant increase in double-positive profiles (i.e., cells co-staining with antibody to BrdU and NeuN) in poststroke animals infused with $\mathrm{CD} 34^{+}$cells (Figure 6D). Consistent with these data, total neuronal counts (Figure 6E) from sections stained with $\mathrm{NeuN}$ in the left cortex 90 days after cell transplantation demonstrated a significant increase in the number of cells visualized in animals transplanted with $\mathrm{CD}_{3} 4^{+}$cells compared with those receiving CD34cells or PBS. In addition, vasculature was visualized in the ischemic zone of the forebrain of animals treated with CD34+ cells 90 days later using anti-mouse CD31 as a marker. A distinct vascular network was seen in the expanded cortex associated with neurogenesis after stroke (Figure 6F). Such vessels displayed a pattern quite distinct from that observed on the contralateral (nonischemic) side (Figure $6 \mathrm{G})$. In these vessels on the ischemic side, some endothelial cells were observed to express human CD31 antigen (Figure 6H). On day 90, mature MAP- $2^{+}$dendrites were observed in the expanded cortex of poststroke animals treated with $\mathrm{CD} 4^{+}$cells (data not shown). These data are consistent with the formation of a stable neovasculature to support regeneration of cortical neurons.

Inhibition and acceleration of angiogenesis by antiangiogenic and angiogenic agents. To investigate the hypothesis that neovascularization is essential to support endogenous neuronal regeneration, we used an antiangiogenic agent, Endostatin. The latter is known to inhibit proliferation of endothelial cells (21) and to have a direct inhibitory effect on EPCs (22). Mice were subjected to stroke, $\mathrm{CD} 4^{+}$or $\mathrm{CD} 34^{-}$cells were administered, and animals were divided into two groups; one group received Endostatin and the other PBS ( $n=5$, for each group). On day 7 after cell transplantation, treatment with Endostatin suppressed endothelial proliferation to $18 \% \pm 3 \%$ and $22 \% \pm 3 \%$ in mice transplanted with CD34- cells and $\mathrm{CD} 34^{+}$cells, respectively, compared with proliferation observed in PBS-treated controls. Administration of Endostatin also impaired cortical expansion due to transplantation of CD $34^{+}$cells. On day 14 , the cortical width index was $0.33 \pm 0.01$ and $0.41 \pm 0.01$ with and without Endostatin, respectively $(P<0.01)$. Animals treated with $\mathrm{CD} 34^{+}$cells and Endostatin after stroke displayed a reduction in migrating NPCs; $101 \pm 7$ cells per high-power field (HPF), for animals treated with $\mathrm{CD} 34^{+}$cells + PBS, and $21 \pm 2$ cells $/$ HPF for animals treated with $\mathrm{CD}_{3} 4^{+}$cells + Endostatin $(P<0.01)$. Similarly, on day 14 , the number of neurons in the poststroke cortex was less in mice treated with CD $34^{+}$cells + Endostatin $(1,469 \pm 53)$, compared with animals treated with CD $34^{+}$cells + PBS $(2,213 \pm 36 ; P<0.01)$.

To gain further support for our hypothesis, we used the proangiogenic agent erythropoietin (EPO). $\mathrm{EPO}$, well known for its essential role in regulating proliferation and differentiation of erythroid cells, has recently been found to promote mobilization of EPCs (23) and to have angiogenic potential (24). On day 4 after stroke, a significant increase in circulating $\mathrm{CD} 34^{+}$cells was observed with EPO injection $(5,244 \pm 1,267$ and 1,333 \pm 389 cells $/ \mathrm{ml}$ in the EPO and PBS groups, respectively; $n=6$ per group, $P<0.05)$. In addition, mice treated with EPO displayed enhanced neovascularization based on angiographic score (5.4 \pm 0.8 and $1.2 \pm 0.4$, in EPO and PBS groups, respectively; $n=5$ per group, $P<0.05)$. On day 28 after stroke, treatment with EPO increased the number of cells co-staining for BrdU and NeuN (3.4 \pm 0.3 and $0.4 \pm 0.1 / \mathrm{HPF}$ in the EPO and PBS groups, respectively; $n=5$ per group, $P<0.05)$. It should be noted that the magnitude of the stimulatory effect of EPO treatment on angiogenesis and neurogenesis in these experiments was less than that observed in studies with $\mathrm{CD}_{3} 4^{+}$cell transplantation, probably because of the greater number of cells transferred in the latter case.

Neurogenesis in 24-week-old mice. To analyze the effect of increased age on accelerated neurogenesis associated with $\mathrm{CD} 34^{+}$cell transplantation, 24-week-old SCID mice were used. Compared with poststroke mice treated with $\mathrm{CD}^{-} 4^{-}$cells (Figure 7A), transplantation of $\mathrm{CD}^{2} 4^{+}$cells (Figure 7B) showed a significant increase in area occupied by the left cortex on day 35 (Figure 7C). In addi- 

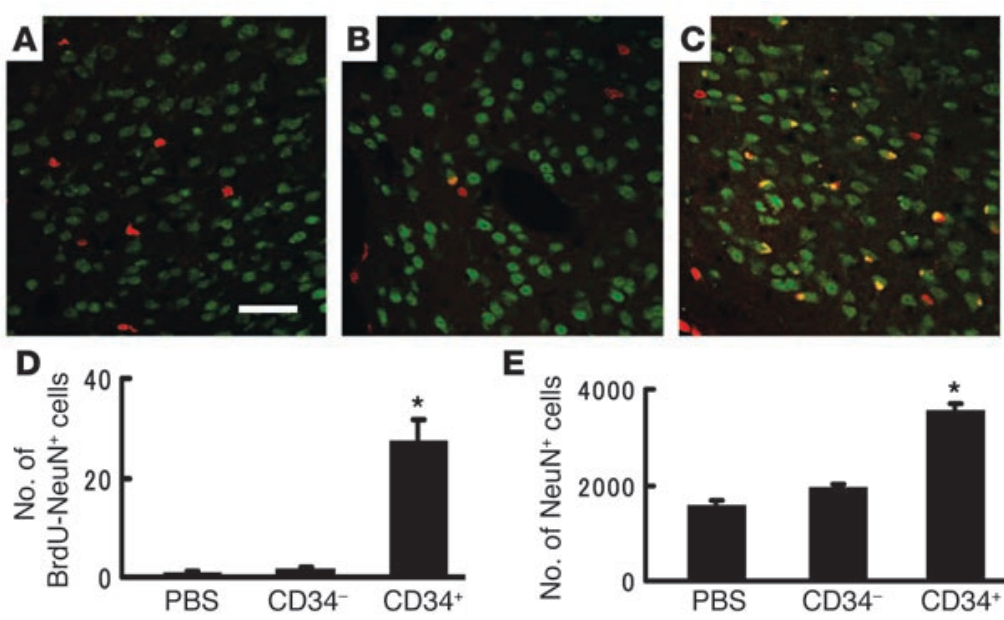

E
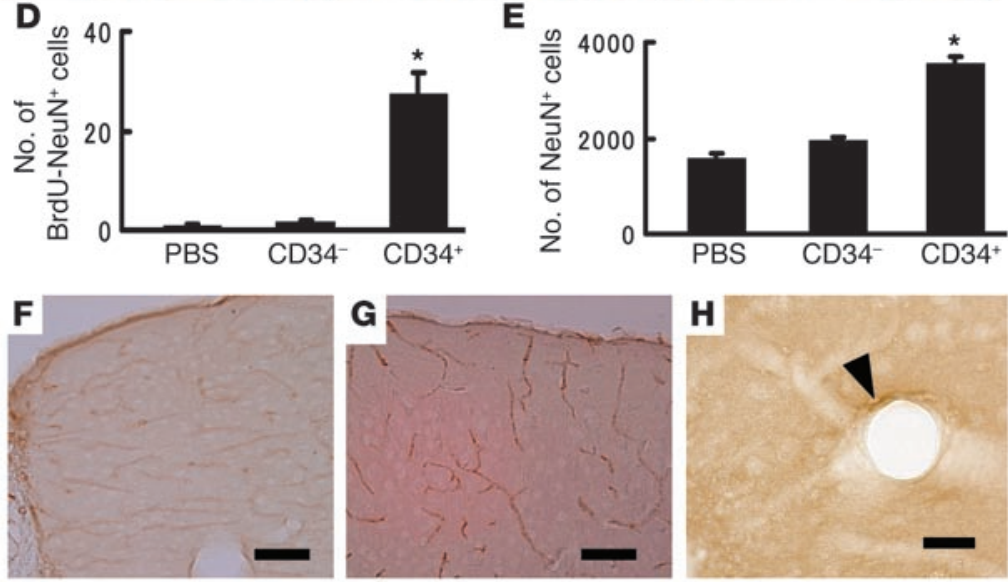

\section{Figure 6}

Therapeutic neovascularization supports survival of regenerating neurons. $(\mathbf{A}-\mathbf{C})$ Animals treated with PBS alone (A), CD34- cells (B), or CD34+ cells (C) were infused with BrdU, killed, and studied immunohistochemically with antibody to BrdU (red), NeuN (green), or both (yellow). (D) The average number of double-positive cells (stained with antibody to BrdU and NeuN) per HPF on day 90 after cell transplantation. Ten fields were evaluated in each animal, and $n=6$ per group. ${ }^{*} P<0.05$ versus PBS. (E) On day 90 after cell transplantation, brain sections from animals treated with PBS alone, CD34- cells, or CD34+ cells were stained with antibody to NeuN, and the number of total neurons in the left cortex was counted. (E) The average number of total $\mathrm{NeuN}^{+}$cells in the left cortex. $n=6$ per group; * $P<0.05$ versus PBS. ( $\mathbf{F}$ and $\mathbf{G}$ ) On day 90 after cell transplantation, mouse CD31 was visualized immunohistologically in forebrain sections from poststroke animals. Newly formed vascular networks were observed in the expanded cortex. The vascular pattern displayed by these neovessels on the ipsilateral $(\mathbf{F}$, ischemic side) was different from that observed on the contralateral side $(\mathbf{G})$. (H) On day 90, human CD31 was visualized immunohistologically. Human endothelial cells were observed in the regenerating cortex of animals treated with CD34+ cells after stroke. The arrowhead shows a human $\mathrm{CD} 31^{+}$endothelial cell. Scale bars: $50 \mu \mathrm{m}(\mathbf{A}), 100 \mu \mathrm{m}$ (F and $\mathbf{G})$, and $20 \mu \mathrm{m}(\mathbf{H})$. tion, evidence of vascular activation, as judged by reactivity with anti-CD13 antibody, was observed in mice treated with CD34 ${ }^{+}$ cells (Figure 7D, CD34- cells; Figure 7E, CD34+ cells). Migration of NPCs was observed in mice treated with CD34- cells (Figure 7F) and $\mathrm{CD} 34^{+}$cells (Figure $7 \mathrm{G}$ ), but a significant enhancement was observed in animals receiving $\mathrm{CD} 34^{+}$cells (Figure $7 \mathrm{H}$ ). Only a thin layer of migrating PSA-NCAM ${ }^{+}$NPCs was observed at the ischemic edge in animals treated with CD34- cells (Figure 7I). In contrast, $\mathrm{CD}^{2} 4^{+}$cell transplantation resulted in a much thicker layer of $\mathrm{PSA}^{-N_{C A M}}{ }^{+}$cells (Figure 7J). Comparing these data in 24-weekold animals with our previous results using 5 -week-old mice, the older animals displayed a similar cortical width index ( 5 weeks old, $0.45 \pm 0.01 ; 24$ weeks old, $0.43 \pm 0.02 ; P>0.05$ ), although older age was associated with a decrease in NPCs migrating into the cortex (5 weeks old, $71 \pm 3$ cells/HPF; 24 weeks old, $43 \pm 4$ cells/HPF; $P<0.05)$ on day 35 after $\mathrm{CD} 34^{+}$cell transplantation.

\section{Discussion}

Our findings show that administration of human $\mathrm{CD} 34^{+}$cells to immunocompromised mice 48 hours after stroke enhances neovascularization at the border of the ischemic zone followed by endogenous neurogenesis. Furthermore, suppression of the neo-
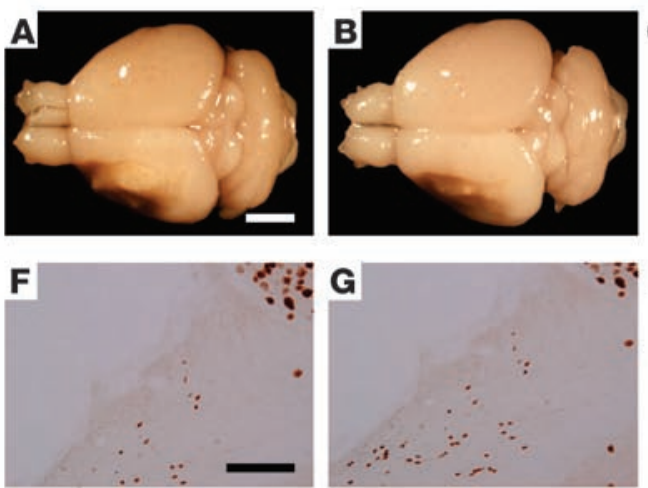
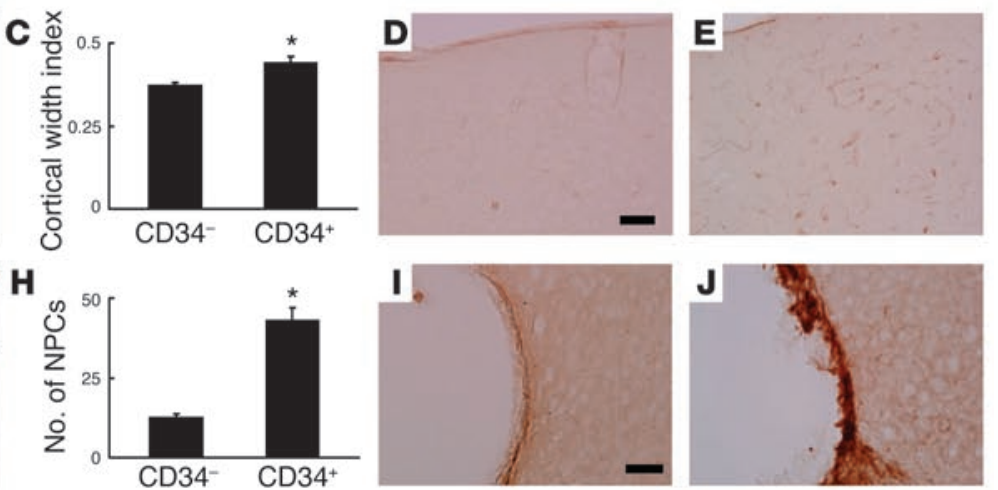

Figure 7

CD34+ cell transplantation in 24-week-old animals. (A-C) On day 35 after cell transplantation, the brains were evaluated. Compared with poststroke mice treated with CD34- cells (A), animals transplanted with CD34+ cells (B) showed an increase in area occupied by the left cortex. Significant cortical regeneration was induced by $\mathbf{C D} 34^{+}$cells transplantation (C). (D and E) Compared with CD34- cell transplantation (D), increased evidence of activated vasculature was observed in animals receiving CD34+ cells $(\mathbf{E})$, as detected with mouse specific anti-CD13 antibody. $(\mathbf{F}-\mathbf{H})$ Migration of NPCs (small NeuN ${ }^{+}$nuclei migrating toward the cortex) was observed in poststroke mice treated with CD34- cells (F) and with CD34+ cells $(\mathbf{G})$. However, a significant increase in migrating NPCs was induced by CD34+ cell transplantation $(\mathbf{H})$. (I and $\mathbf{J})$ A thin layer of migrating PSA-NCAM+ NPCs was observed at the ischemic edge of the cortex in animals treated with CD34- cells (I), compared with a much thicker layer in those receiving CD34+ cells $(\mathbf{J})$. Scale bars: $2 \mathrm{~mm}(\mathbf{A}), 0.1 \mathrm{~mm}(\mathbf{D}$ and $\mathbf{I})$, and $0.4 \mathrm{~mm}(\mathbf{F}) . n=4$ in each group; ${ }^{*} P<0.05$ versus CD34- cells. 
vascularization by an antiangiogenic agent impaired neurogenesis. On the basis of these data, accelerated neovessel formation seems to be essential for enhancing endogenous neurogenesis and improving functional recovery.

The generation of new neurons in the adult is largely restricted to two regions: the SVZ lining the lateral ventricles and the subgranular zone of the dentate gyrus (25). In the transient cerebral ischemia model, evidence has been provided that neuronal regeneration occurs $(10,20)$. However, even in a transient ischemia model in which integrity of the microcirculation is maintained, it was shown that greater than $80 \%$ of newly formed neurons died, most likely because of unfavorable environmental conditions including lack of trophic support and exposure to products of damaged tissue. These considerations may underlie the observation that only $0.2 \%$ of nonviable ischemic neurons were replaced through neurogenesis (20). Such previous observations are consistent with our current results in animals treated with CD34- cells or PBS, in which there was no enhancement of neovascularization, no neurogenesis, and no functional recovery.

Consistent with these data, Endostatin-mediated suppression of endothelial proliferation and direct effects on EPCs abrogated the beneficial effect $\mathrm{CD} 34^{+}$cells on neurogenesis and cortical expansion, and at the same time inhibited formation of neovasculature after stroke. In contrast, when EPO was used as a proangiogenic agent (recently, EPO has also been found to promote mobilization of EPCs) $(23,24)$, accelerated formation of neovasculature was accompanied by enhanced neurogenesis after stroke in our murine model. Our data provide the first direct link between therapeutic neovascularization after stroke and enhanced neurogenesis; formation of neovasculature after stroke supported neurogenesis. Consistent with a previous report (26), activation of NPCs after stroke was induced in adult, as well as young, murine brain. The number of activated NPCs was less in the adult animals, but a significant increase was still observed consequent to $\mathrm{CD} 34^{+}$cells transplantation, with evidence of activated vasculature in brains of the older animals. These findings support our hypothesis that administration of $\mathrm{CD} 34^{+}$cells provides a milieu favorable for neovascularization and endogenous neurogenesis, even in the mature brain.

A relationship between angiogenesis and neurogenesis would be consistent with regeneration of parenchymal cells in other organs subject to therapeutic angiogenesis $(27,28)$. Mechanisms underlying this observation might include more optimal preparation of the ischemic tissue bed for neuronal regeneration by accelerated removal of debris and toxic products, and/or the enhanced production of chemokines and trophic agents by neovasculature. Factors involved might be FGF2 (11), PDGF (29), brain-derived neurotrophic factor (30), and IL-8 (31), which have the capacity to induce mitogenesis, differentiation, recruitment, and survival of NPCs and newly generated neurons. In addition, mediators produced by $\mathrm{CD} 34^{+}$cells (4), such as VEGF, FGF2, and IGF-1, have been shown to accelerate endogenous neurogenesis (10-12). The effects of these and other factors derived from $\mathrm{CD} 34^{+}$cells $(32,33)$ acting on the vasculature are also likely to have an important role in providing an environment conducive to neurogenesis. Newly formed neurons in the setting of neovascularization have been shown to integrate into neuronal networks in adult animals (34); in fact, lack of participation of neurons in such neuronal circuits is probably associated with cell death (20). Our results provide strong support for the hypothesis that neovascularization consequent to administration of $\mathrm{CD} 34^{+}$cells induces neurogenesis by providing the necessary supportive environment. Whereas perturbation of the neurovascular unit has been proposed to contribute to tissue dam- age in stroke, neoangiogenesis and accompanying neurogenesis could be considered to be rebuilding crucial elements of the neurovascular unit (35). Previous reports have demonstrated that endothelial cells in newly formed vessels after stroke mainly originate from pre-existing endothelial cells (due to proliferation) with a contribution of circulating EPCs $(36,37)$. Consistent with these findings, we observed proliferating mouse endothelial cells around the ischemic area soon after stroke. The latter endothelial cells could be visualized, at least in part, with antibody specific for human CD31, after transplantation of human $\mathrm{CD}_{3}{ }^{+}$cells. Furthermore, diminished neovascularization was observed after stroke following transplantation of CD34 $/ \mathrm{Flk}-1^{-}$cells, compared with $\mathrm{CD} 34^{+}$cells (containing both Flk- $1^{-}$and Flk- $1^{+}$cells) despite similar activation of murine endothelial cells in the ischemic territory in each case. These results support potential contribution of $\mathrm{CD} 34^{+} / \mathrm{Flk}-1^{+}$cells (a population known to be rich in EPCs) in expansion of the vascular network after stroke.

The actual pattern of the newly formed vascular network was quite different on the ipsilateral (ischemic) from the contralateral side in poststroke mice treated with $\mathrm{CD} 34^{+}$cells. This observation suggests that formation of vasculature consequent to transplantation of CD $34^{+}$ cells after stroke does not simply reconstitute the original vascular network. Rather, a new vascular pattern arises (a true neovasculature) that is capable of supporting neurogenesis, followed by functional recovery, even though it displays a relatively "aberrant" pattern, at least anatomically, compared with the contralateral vasculature.

Our observations provide evidence of a crucial role for neovessel formation, achieved through the administration of CD $34^{+}$cells after stroke, in processes that underlie neurogenesis. These data strongly suggest that neovascularization is essential for neuronal regeneration after stroke and that therapeutic neovascularization is a potentially effective means of enhancing functional recovery. Our observations might explain the mild therapeutic effect achieved by neuronal cell transplantation after stroke reported in humans (38). This leads to the hypothesis that therapeutic neovascularization may be required to achieve optimal "take" of transplanted neuronal precursors in the setting of ischemia. Although the current data bear most directly on the endogenous neuronal response to cerebrovascular ischemia, it is possible that enhanced formation of neovasculature may also be important for survival of embryonic (39) and neural (40) stem cell transplants in other circumstances, such as neurodegenerative disorders.

\section{Methods}

All procedures were done in accordance with the National Cardiovascular Center Animal Care and Use, and Human Assurance Committees. Quantitative measurements and behavioral tests were conducted by investigators blinded to the experimental protocol and identity of the sections and animals under study.

Induction of focal cerebral ischemia. Permanent focal cerebral infarction was produced by ligation and disconnection of the distal portion of the left MCA. Male SCID mice (5 weeks old; Oriental Yeast Co. Ltd., Tokyo, Japan) were used for experiments involving human cell transplantation. Male C57BL/6J mice (24 weeks old; Clea Japan Inc., Tokyo, Japan) were used for experiments with human recombinant EPO (Kirin, Tokyo, Japan). Under halothane inhalation (3\%), the left MCA was isolated, electrocauterized, and disconnected just distal to its crossing of the olfactory tract (distal M1 portion). $\mathrm{CBF}$ in the MCA area was monitored as described (41). Mice that showed decreased CBF by approximately $75 \%$ immediately after and 24 hours after ligation were used for our experiments (success rate of $>95 \%$ ). Body temperature was maintained at $36.5-37^{\circ} \mathrm{C}$ using a heat lamp during 
the operation and for 2 hours after MCA occlusion. The ratio of CBF after cell transplantation to $\mathrm{CBF}$ before cell transplantation was calculated to evaluate the change of $\mathrm{CBF}$ (termed relative $\mathrm{CBF}$ ).

Analysis of perfusion and brain infarction after MCA occlusion. Carbon black ink (Fuekinori Kogyo Co. Ltd., Osaka, Japan) was used to delineate the perfused area after MCA ligation as described (42). Viability of brain tissue was evaluated using TTC (Sigma-Aldrich, St. Louis, Missouri, USA). Coronal sections ( $1 \mathrm{~mm}$ thick) of the forebrain were stained with $1 \%$ TTC, and the images of sections from the exact center of the forebrain were captured using a microscopic digital camera system (Olympus, Tokyo, Japan). The width of viable cortex was determined by NIH image.

Administration of $\mathrm{CD} 34^{+}$cells after stroke. $\mathrm{CD} 34^{+}$cells (containing Flk-1- $1^{-}$and Flk- $1^{+}$cell populations) and CD34 $4^{+} / \mathrm{Flk}-1^{-}$cells were isolated from cord blood using a Direct CD34 Progenitor Cell Isolation kit (Miltenyi Biotec, Bergisch Gladbach, Germany), CD34 Multisort kit (Miltenyi Biotec), and anti-Flk-1 antibody (Sigma-Aldrich), according to the manufacturers' protocols. CD34mononuclear cells were also collected for control experiments. In each case, cell populations were analyzed by FACS (BD Biosciences, San Jose, California, USA) using PerCP-conjugated anti-CD34 antibody (BD Biosciences), phycoerythrinconjugated (PE-conjugated) anti-P1H12 antibody (BD Biosciences), and anti-Flk-1 antibody (Sigma-Aldrich) conjugated with FITC according to the manufacturer's protocol (Zenon Alexa Fluor; Molecular Probes Inc., Eugene, Oregon, USA). For labeling studies, cells were incubated with the fluorescent dye chloromethylbenzamido (CM-DiI; Molecular Probes Inc.). Forty-eight hours after stroke, $5 \times 10^{5} \mathrm{CD} 34^{+}, \mathrm{CD} 34^{+} / \mathrm{FLK}^{-} 1^{-}, \mathrm{CD} 34^{-}$cells, or the same volume $(100 \mu \mathrm{l})$ of PBS were infused intravenously via the tail vein.

For the analysis of the effect of aging on neurogenesis, 24-week-old male SCID mice were used. Because of the known increased evidence of tumors, infection (for example, excess mucus in the eye), and elevated plasma immunoglobulins in older SCID mice, the presence of each of these conditions was also investigated. Mice with evidence of weight loss, tumors, infection, or elevated plasma immunoglobulins ( $>10 \mu \mathrm{g} / \mathrm{ml}$ ) were excluded from further analysis.

Immunohistochemistry. Mice brains were removed and fixed in paraformaldehyde. Coronal sections $(20 \mu \mathrm{m})$ were prepared using a vibratome (Leica Microsystems Inc., Wetzlar, Germany). To assess cell proliferation and angiogenesis, fresh frozen sections $(20 \mu \mathrm{m})$ were prepared and fixed in acetone. Sections were subjected to immunohistochemistry with antibody to NeuN (Chemicon International, Temecula, California, USA), mouse-specific antibody to CD31 (BD Biosciences), human-specific antibody to CD31 (DAKO A/S, Glostrup, Denmark), mouse-specific antibody to CD13 (Santa Cruz Biotechnology, Santa Cruz, California, USA), and antibodies to MAP-2 (Sigma-Aldrich), human von Willebrand Factor (DAKO A/S), PSA-NCAM (Chemicon International), DCX (Chemicon International), Musashi-1 (19), TUNEL (Molecular Probes Inc.), or BrdU (Boehringer Ingelheim, Ingelheim, Germany).

Cell proliferation analysis. For assessment of cell proliferation in vivo, BrdU (Sigma-Aldrich) was administered intraperitoneally. In the case of endothelial cell proliferation, BrdU $(200 \mathrm{mg} / \mathrm{kg})$ was administered 24 hours before killing. For endogenous neurogenesis, $50 \mathrm{mg} / \mathrm{kg}$ of BrdU was injected every second day for a total of 14 days. Cells co-staining for BrdU and mousespecific antibody to CD31 using confocal microscopy (Olympus) in HPFs ( $\times 40$ of objective lens) were counted as proliferating endothelial cells. The cell population co-staining for BrdU and NeuN was counted as regeneratingproliferating neurons. For quantitative analysis, brain sections at the exact center of the forebrain were stained, and the left cortical area of $1 \mathrm{~mm}$ (or >1 $\mathrm{mm}$ ) distal from the midline was examined by two investigators blinded to the experimental protocol. In each case, 10 representative fields were evaluated before quantitative results were analyzed.

Assessment of angiogenesis. For assessment of angiogenesis at the border of MCA and ACA areas, carbon black perfusion was used to visualize the vasculature and TTC staining to demarcate the border of viable and nonviable tissue. Semi-quantitative analysis of angiogenesis made use of the angiographic score and was calculated by a previously described method (43). Microscopic digital images were scanned into a computer (Olympus), and an overlay, composed of 50- $\mu \mathrm{m}$-diameter circles arranged in rows spaced $100 \mu \mathrm{m}$ apart, was superimposed on the image. The total number of grids intersecting the border zone containing a microvessel was counted. Mousespecific antibody to CD13 was used to visualize 'activated' vasculature.

Assessment of ischemic brain damage and neurogenesis. For determination of the cortical width index, whole-brain images were captured using a microscopic digital camera system (Olympus). The width at the midpoint of the forebrain was measured, and the ratio of left width to right width was defined as the cortical width index. To quantify accelerated neurogenesis, three brain sections around the center of the forebrain on day 14 after cell transplantation were stained with NeuN or TUNEL. The number of small NeuN nuclei-positive cells in the white matter at lower left of the left cortex and the number of TUNEL ${ }^{+}$cells around the lower part of ischemic cortical edge was counted in HPFs. For determining the number of neurons in the left cortex, brain sections of the exact center of the forebrain on day 90 were stained with $\mathrm{NeuN}$, and the total number of $\mathrm{NeuN}^{+}$cells was quantitated.

Behavioral analysis. To assess cortical function, mice were subjected to behavioral testing in the open-field task (44) on day 35 or 90 after cell transplantation. Auditory startling behavior was tested as described (45). To estimate learning activity, mice were tested by water maze test (46) and passive avoidance test (47). To exclude the contribution of physical deficits directly related to the operative procedure and induction of stroke, motor deficiencies were examined on days 9 and 16 after stroke. Neurological deficits were scored on a three-point modified scale as described (17). Body weight, monitored in each experimental group, displayed no significant differences (data not shown).

Inbibition and promotion of neovascularization. Inhibition of neovascularization was achieved by administering Endostatin (10 $\mu \mathrm{g}$; subcutaneous; Calbiochem-Novabiochem, Darmstadt, Germany) once daily for 14 days. To stimulate mobilization of EPCs originating in the host, EPO $(1,000$ $\mu \mathrm{g} / \mathrm{kg}$ ) was injected subcutaneously 24,48 , and 72 hours after ischemia. Peripheral blood cells were analyzed using FITC-conjugated anti-mouse CD45 (BD Biosciences), PE-conjugated anti-mouse CD34 antibodies (BD Biosciences), and 7-amino-actinomycin D viability Dye (Beckman Coulter Inc., Marseille Cedex, France), as described (48), using counting beads (BD Biosciences) as an internal control.

Data analysis. In all experiments, mean \pm SE is reported. Statistical comparisons among groups were determined using one-way ANOVA. Where indicated, individual comparisons were performed using Student's $t$ test.

\section{Acknowledgments}

Musashi-1 was generously provided by H. Okano at Keiou University in Japan. This work was partially supported by the Uehara, Takeda, and Terumo Medical Foundations, Grant-in-Aid for Scientific Research from the ministry of Education (15590785) and from the Ministry of Health, Labour, and Welfare (16C-7, H16-CK-004). We would like to thank Y. Kasahara, K. Obata, and Y. Okinaka for technical assistance.

Received for publication November 24, 2003, and accepted in revised form May 18, 2004.

Address correspondence to: Akihiko Taguchi, Department of Cerebrovascular Disease, National Cardiovascular Center, 5-7-1 Fujishiro-dai, Suita, Osaka 565-8565, Japan. Phone: 81-6-68335012; Fax: 81-6-6872-7485; E-mail: ataguchi@res.ncvc.go.jp. 
1. Asahara, T., et al. 1997. Isolation of putative progenitor endothelial cells for angiogenesis. Science. 275:964-967.

2. Asahara, T., et al. 1999. Bone marrow origin of endothelial progenitor cells responsible for postnatal vasculogenesis in physiological and pathological neovascularization. Circ. Res. 85:221-228.

3. Kawamoto, A., et al. 2001. Therapeutic potential of ex vivo expanded endothelial progenitor cells for myocardial ischemia. Circulation. 103:634-637.

4. Majka, M., et al. 2001. Numerous growth factors, cytokines, and chemokines are secreted by human $\mathrm{CD}_{3} 4^{+}$cells, myeloblasts, erythroblasts, and megakaryoblasts and regulate normal hematopoiesis in an autocrine/paracrine manner. Blood. 97:3075-3085

5. Taguchi, A., Ohtani, M., Soma, T., Watanabe, M., and Kinosita, N. 2003. Therapeutic angiogenesis by autologous bone-marrow transplantation in a general hospital setting. Eur. J. Vasc. Endovasc. Surg. 25:276-278

6. Tateishi-Yuyama, E., et al. 2002. Therapeutic angiogenesis for patients with limb ischaemia by autologous transplantation of bone-marrow cells: a pilot study and a randomised controlled trial. Lancet. 360:427-435.

7. Hamano, K., et al. 2001. Local implantation of autologous bone marrow cells for therapeutic angiogenesis in patients with ischemic heart disease: clinical trial and preliminary results. Jpn. Circ.J. 65:845-847.

8. Chen, J., et al. 2001. Intravenous administration of human umbilical cord blood reduces behavioral deficits after stroke in rats. Stroke. 32:2682-2688.

9. Hess, D.C., et al. 2002. Bone marrow as a source of endothelial cells and NeuN-expressing cells after stroke. Stroke. 33:1362-1368.

10. Nakatomi, H., et al. 2002. Regeneration of hippocampal pyramidal neurons after ischemic brain injury by recruitment of endogenous neural progenitors. Cell. 110:429-441.

11. Drago, J., Murphy, M., Carroll, S.M., Harvey, R.P., and Bartlett, P.F. 1991. Fibroblast growth factormediated proliferation of central nervous system precursors depends on endogenous production of insulin-like growth factor I. Proc. Natl. Acad. Sci. U. S. A. 88:2199-2203.

12. Jin, K., et al. 2002. Vascular endothelial growth factor (VEGF) stimulates neurogenesis in vitro and in vivo. Proc. Natl. Acad. Sci. U. S. A. 99:11946-11950.

13. Solovey, A., et al. 1997. Circulating activated endothelial cells in sickle cell anemia. N. Engl. J. Med. 337:1584-1590.

14. Bhagwat, S.V., Petrovic, N., Okamoto, Y., and Shapiro, L.H. 2003. The angiogenic regulator CD13/ APN is a transcriptional target of Ras signaling pathways in endothelial morphogenesis. Blood. 101:1818-1826.

15. Van Dam, D., et al. 2003. Age-dependent cognitive decline in the APP23 model precedes amyloid deposition. Eur. J. Neurosci. 17:388-396.

16. Farkas, T., et al. 2003. Peripheral nerve injury influences the disinhibition induced by focal ischaemia in the rat motor cortex. Neurosci. Lett. 342:49-52.

17. Tamatani, M., et al. 2001. ORP150 protects against hypoxia/ischemia-induced neuronal death. Nat. Med. 7:317-323.

18. Iwai, M., et al. 2003. Temporal profile of stem cell division, migration, and differentiation from subventricular zone to olfactory bulb after transient forebrain ischemia in gerbils. J. Cereb. Blood Flow Metab. 23:331-341.

19. Sakakibara, S., et al. 1996. Mouse-Musashi-1, a neural RNA-binding protein highly enriched in the mammalian CNS stem cell. Dev. Biol. 176:230-242.

20. Arvidsson, A., Collin, T., Kirik, D., Kokaia, Z., and Lindvall, O. 2002. Neuronal replacement from endogenous precursors in the adult brain after stroke. Nat. Med. 8:963-970.

21. O'Reilly, M.S., et al. 1997. Endostatin: an endogenous inhibitor of angiogenesis and tumor growth. Cell. 88:277-285.

22. Capillo, M., et al. 2003. Continuous infusion of endostatin inhibits differentiation, mobilization, and clonogenic potential of endothelial cell progenitors. Clin. Cancer Res. 9:377-382.

23. Bahlmann, F.H., et al. 2004. Erythropoietin regulates endothelial progenitor cells. Blood. 103:921-926.

24. Jaquet, K., Krause, K., Tawakol-Khodai, M., Geidel, S., and Kuck, K.H. 2002. Erythropoietin and VEGF exhibit equal angiogenic potential. Microvasc. Res. 64:326-333.

25. Gage, F.H. 2000. Mammalian neural stem cells. Science. 287:1433-1438.

26. Yagita, Y., et al. 2001. Neurogenesis by progenitor cells in the ischemic adult rat hippocampus. Stroke. 8:1890-1896

27. Ross, M.A., Sander, C.M., Kleeb, T.B., Watkins, S.C., and Stolz, D.B. 2001. Spatiotemporal expression of angiogenesis growth factor receptors during the revascularization of regenerating rat liver. Hepatology. 34:1135-1148.

28. Toda, S., et al. 1999. Immunohistochemical expression of growth factors in subacute thyroiditis and their effects on thyroid folliculogenesis and angiogenesis in collagen gel matrix culture. J. Pathol. 188:415-422.

29. Johe, K.K., Hazel, T.G., Muller, T., Dugich-Djordjevic, M.M., and McKay, R.D. 1996. Single factors direct the differentiation of stem cells from the fetal and adult central nervous system. Genes Dev. 10:3129-3140.

30. Leventhal, C., Rafii, S., Rafii, D., Shahar, A., and Goldman, S.A. 1999. Endothelial trophic support of neuronal production and recruitment from the adult mammalian subependyma. Mol. Cell. Neurosci. 13:450-464.

31. Araujo, D.M., and Cotman, C.W. 1993. Trophic effects of interleukin- $4,-7$ and -8 on hippocampal neuronal cultures: potential involvement of glialderived factors. Brain Res. 600:49-55.

32. Valable, S., et al. 2003. Angiopoietin-1-induced PI3-kinase activation prevents neuronal apoptosis. FASEB J. 17:443-445.
33. Sun, W., Funakoshi, H., and Nakamura, T. 2002. Localization and functional role of hepatocyte growth factor (HGF) and its receptor c-met in the rat developing cerebral cortex. Brain Res. Mol. Brain Res. 103:36-48.

34. Louissaint, A., Jr., Rao, S., Leventhal, C., and Goldman, S.A. 2002. Coordinated interaction of neurogenesis and angiogenesis in the adult songbird brain. Neuron. 34:945-960.

35. Lo, E.H., Dalkara, T., and Moskowitz, M.A. 2003. Mechanisms, challenges and opportunities in stroke. Nat. Rev. Neurosci. 4:399-415.

36. Beck, H., et al. 2003. Participation of bone marrow-derived cells in long-term repair processes after experimental stroke. J. Cereb. Blood Flow Metab. 23:709-717.

37. Zhang, Z.G., Zhang, L., Jiang, Q., and Chopp, M. 2002. Bone marrow-derived endothelial progenitor cells participate in cerebral neovascularization after focal cerebral ischemia in the adult mouse. Circ. Res. 90:284-288.

38. Kondziolka, D., et al. 2000. Transplantation of cultured human neuronal cells for patients with stroke. Neurology. 55:565-569.

39. Hoehn, M., et al. 2002. Monitoring of implanted stem cell migration in vivo: a highly resolved in vivo magnetic resonance imaging investigation of experimental stroke in rat. Proc. Natl. Acad. Sci. U. S. A. 99:16267-16272.

40. Abe, K. 2000. Therapeutic potential of neurotrophic factors and neural stem cells against ischemic brain injury. J. Cereb. Blood Flow Metab. 20:1393-1408.

41. Matsushita, K., et al. 1998. Marked, sustained expression of a novel $150-\mathrm{kDa}$ oxygen-regulated stress protein, in severely ischemic mouse neurons. Brain Res. Mol. Brain Res. 60:98-106.

42. Matsuyama, T., et al. 1983. Why are infant gerbils more resistant than adults to cerebral infarction after carotid ligation? J. Cereb. Blood Flow Metab. 3:381-385.

43. Takeshita, S., et al. 1994. Therapeutic angiogenesis. A single intraarterial bolus of vascular endothelial growth factor augments revascularization in a rabbit ischemic hind limb model. J. Clin. Invest. 93:662-670.

44. Kimble, D.P. 1968. Hippocampus and internal inhibition. Psychol. Bull. 70:285-295.

45. Sasaki, H., Iso, H., Coffey, P., Inoue, T., and Fukuda, Y. 1998. Prepulse facilitation of auditory startle response in hamsters. Neurosci. Lett. 248:117-120.

46. Balschun, D., et al. 2003. Does cAMP response element-binding protein have a pivotal role in hippocampal synaptic plasticity and hippocampusdependent memory? J. Neurosci. 23:6304-6314.

47. Mereu, G., et al. 2003. Prenatal exposure to a cannabinoid agonist produces memory deficits linked to dysfunction in hippocampal long-term potentiation and glutamate release. Proc. Natl. Acad. Sci. U. S. A. 100:4915-4920.

48. Gratama, J.W., et al. 1999. Comparison of singleand dual-platform assay formats for $\mathrm{CD} 34^{+}$haematopoietic progenitor cell enumeration. Clin. Lab. Haematol 21:337-346. 